\title{
Primary Intra-Osseous Squamous Cell Carcinoma of the Mandible - A Case Report
}

\author{
Dr. Ruchika Garg ${ }^{1}$, Dr. Anjana S Bagewadi ${ }^{2}$, Dr. Sidramesh Muttagi ${ }^{3}$ \\ ${ }^{1,2,3}$ (Department of Oral medicine and radiology, KLE VKIDS, KLE University)
}

\begin{abstract}
Primary intra-osseous squamous cell carcinoma (PIOSCC) is a rare malignant tumor of the jaw. It is more common in adults, in six to seven decades of life with a male to female ratio of 3:1 and predominantly located in posterior mandible. Its etiology is not clear however the most common factor may be a reactive inflammatory stimulus with or without genetic predisposition. We hereby report a case of PIOSCC involving the mandible of a 48-year-old female. The absence of any defect, proliferative growth or mass intraorally suggested that the origin of the squamous cells was de novo from within the bone. Understanding of the clinical, radiographic, and histopathological features of this tumor allows accurate diagnosis and appropriate treatment of this rare malignancy.
\end{abstract}

Keywords: A Primary intra-osseous squamous cell carcinoma, mandible, jaw

\section{Case Report}

A 48-year-old female reported to our department with a complaint of pain and swelling on the left side of lower one-third of the face since 3 months. She visited the dentist for the first time. She reported difficulty in mouth opening with no pus discharge or bleeding from the lesion. There was no relevant medical and family history. Nevertheless, the patient gave a history of tobacco chewing since 8 years, thrice/day.

Extra-oral examination revealed a solitary lymph node which was present in the left sub-mandibular region which was palpable, firm in consistency and non-tender. A large and firm swelling was present in the left mandibular region measuring about $5 \mathrm{~cm} \times 6 \mathrm{~cm}$ causing slight asymmetry in the left side of the face. The swelling was diffuse involving middle and lower third of the left side of the face extending from ala tragal line to $2 \mathrm{~cm}$. below the inferior border of mandible supero-inferiorly. The swelling extended from angle of mouth to the posterior border of ramus of mandible antero-posteriorly [Fig. 1]. Paresthesia was present in relation to left side of the lower lip. Mouth opening was reduced to $2 \mathrm{~cm}$ without deflection or deviation of mandible. On palpation, swelling was non-tender and firm in consistency without any local rise in temperature.

Intra-oral examination revealed a Class I molar relation without any occlusal derangement. A diffuse swelling was present in the right buccal vestibule measuring about $1 \mathrm{X} 1 \mathrm{~cm}$. in dimension in relation to teeth 35,36. It was soft in consistency and tender on palpation. No obliteration of buccal vestibule was seen [Fig. 2]. Patient had undergone extraction 4 months ago for 37. Grade II mobility was seen in relation to 34, 35 and 36. The alveolar socket of 37,38 had completely healed with overlying mucosa appearing normal.

\section{Investigations}

Patient was advised for serology test for HIV 1\&2, IOPA, orthopantogram (OPG) and Computed Tomography (CT). The immunological status of the patient was normal as she was seronegative for HIV $1 \& 2$. IOPA of 36 region showed an ill-defined radiolucency extending from the mesial aspect of 35 to the region posterior to 36 and extending from the periapical region of 35,36 to the inferior border of mandible with no break in continuity in the inferior border of mandible [Fig. 3].

OPG showed a large ill-defined radiolucency measuring about $7 \times 8 \mathrm{~cm}$ in dimension and extending from the mesial aspect of 34 to the ramus of mandible antero- posteriorly and from the sigmoid notch involving the condyle and coronoid process to the inferior border of mandible supero-inferiorly. The borders of the radiolucency exhibited an irregularity. The internal structure was not homogeneous and there were mixed radiolucent and radio-opaque areas along with presence of bay within bay appearance which is a classical feature of malignancy [Fig. 4].

CT revealed a destructive lesion in the left half of the mandible extending from subcondylar to right ramus and angle of mandible. It showed a unicentric lesion with inside out growth and permeative type of destruction. The margins were indistinct with wide zone of transition suggesting an intraosseous malignant neoplasm. Masseter and part of pterygoid muscle was bulky suggesting infiltration of the lesion. [Fig. 5,6]. 


\section{Differential Diagnosis}

On the basis of history, clinical findings and radiographs, a differential diagnosis of chronic suppurative osteomyelitis, malignancy which is primary in nature ( For eg. Epithelial in origin - Squamous cell carcinoma, intra-osseous carcinoma or Connective tissue in origin like Sarcomas), secondary malignancy (metastasis) was given. Chronic suppurative osteomyelitis is a condition which causes inflammation of the bone marrow and is characterized by pus production initially and formation of calcified components later on. The etiology is usually an odontogenic infection or a fracture following trauma. It has a more predilection for men than women and mostly occurs in mandibular premolar region. Radiographically, it is charactrized by a poorly defined radiolucency which is irregular and gives a moth-eaten appearance and the presence of sequestrum. In the present case, there was a history of extraction of teeth and clinical features like trismus, paresthesia and mobility of the remaining teeth could lead to diagnosis of suppurative osteomyelitis. However, the absence of pus discharge or sinus opening on intra-oral examination and absence of sequestrum in radiograph, helped in ruling out this condition.

Squamous cell carcinoma is the most common malignant tumor of the oral cavity. It has two types of origin, it can arise from the epithelium of oral cavity or de novo from within the bone from the epithelial cell rests or due to malignant transformation of the epithelium of odontogenic lesions like cysts and ameloblastoma. PIOC is on the other hand not a very common entity.

Sarcomas which arise within the jaw bones include most commonly osteosarcoma and chondrosarcoma. Chondrosarcomas are rarely found in jaws and mostly occurs in the alveolar process of anterior maxilla and at angle and alveolar ridge of premolar-molar region of the mandible. However, it is characterized by its slow growth and painless nature in its early stages. Osteosarcoma is the most common primary malignant tumor of bone and among the jaw bones occur most commonly in the body of mandible. However, it is rare in jaw bones accounting for only $7 \%$ among all osteosarcomas with most frequent association in long bones. It grows rapidly giving a moth-eaten appearance in initial osteolytic stage. The mean age of occurrence is 33 years.

A bone biopsy was performed from 37, 38 region and was sent for histopathological examination. TNM staging of the lesion was T4b N1 M0. Biopsy revealed presence of dysplastic features like keratin pearl formation, cellular nuclear pleomorphism, altered nuclear cytoplasmic ratio, nuclear hyperchromatism, atypical mitotic figures, individual cell keratinization and areas of nerve and vascular invasion. The histopathological features were suggestive of well-differentiated SCC with advancing front showing moderate differentiation [Fig. 7].

The origin of squamous cells can be from the oral mucosa or from within the bone. The absence of any defect, proliferative growth or mass intraorally suggested that the origin of the squamous cells was de novo from within the bone. Hence, a final diagnosis of "PIOC" was considered.

A bone scan was advised to rule out metastasizing lesion, but due to financial constraints of the patient, it was not performed. In view of extension of the tumor into the infratemporal fossa and its abbutment to the spheniod bone (skull base) it was inoperable (T4b N1 M0). Thus palliative chemotherapy was planned.

\section{Discussion}

PIOC describes the SCC that develops likely from the residues of the odontogenic epithelium entrapped within the jaw with no connection with the surface oral mucosa. This tumor was first described by Loos in 1913. The World Health Organization in 1972 proposed the term PIOC and classified this entity as an odontogenic carcinoma[1].

PIOC is more common in adults, in sixth to seventh decade of life with a male to female ratio of 3:1. It is usually situated in the posterior mandible [2]. Its etiology is not clear however the most common factor may be a reactive inflammatory stimulus with or without genetic predisposition [3]. Since SCCs may appear within the bone thus the diagnosis of PIOC is by exclusion. Suei et al. [4] anticipated few diagnostic criteria for PIOC:

1. To differentiate PIOCs from SCCs of surface mucosal origin, no ulcer formation must be present on the overlying oral mucosa except due to trauma or tooth extractions.

2. To exclude the possibility of other odontogenic carcinomas, several sections of the histological specimens should demonstrate SCC without any cystic components or other odontogenic tumor cells.

3. To discard a distant metastasis from a primary tumor, chest radiographs must be evaluated at the time of diagnosis and throughout the follow-up period of more than 6 months. Ideally a PET CT is advisable, if not PET CT, then USG abdomen, mammogram, chest radiograph are advisable. Chest Radiograph was within normal limits in this patient.

In our case, the mucosa overlying the tumor and the surrounding mucosa were quite normal. The first and the most common clinical features may be pain and swelling of the affected area. In a study conducted by 
Thomas et al.3 on 33 patients pain was the most common presenting feature in $17(54.8 \%)$ patients followed by swelling of the jaw in $16(51.6 \%)$ and sensory disturbances were reported in five cases $(16.1 \%)$.

The PIOCs of the jaws are classified based on their possible origins into four types:

Type 1:

PIOC from odontogenic cysts.

Type 2:

A: Malignant ameloblastoma

B: Ameloblastic carcinoma

Type 3:

PIOC arising de novo $\bullet$ Keratinizing $\bullet$ Nonkeratinizing

Type 4:

Intra osseous mucoepidermoid carcinoma[5].

The radiological investigations provide valuable information in diagnosing these clinically perplexing conditions. PIOC exhibit radiolucency with a wide variation in size and shape. Thomas et al. in his study reported varied radiographic presentations like small radiolucent loculations, well-defined lesions and poorly defined moth-eaten appearance[6]. Slowly growing tumors often display well-defined peripheries, whereas rapidly expanding lesions exhibit poorly defined, ragged borders with permeative type of destruction. Degree of raggedness of the border may reflect the aggressiveness of the lesion. Pathological fracture occurs due to cortical plate thinning with subsequent step deformity. The internal structure is totally radiolucent with very little residual bone left within the center of the lesion. Root resorption is unusual. Teeth that lose both lamina dura and the supporting bone appear to be "floating" in space. If the lesions are not aggressive, they may be mistaken for periapical cysts and granulomas. If the lesions are infiltrative with extensive bone destruction, a metastatic lesion must be excluded as well as multiple myelomas, fibrosarcoma and carcinoma arising in a dental cyst must be ruled out.

Histologically, they vary from well-differentiated tumors exhibiting keratinization to nonkeratinized poorly differentiated carcinomas. Yamada et al. in his clinicopathologic study of five cases of PIOC found three cases of well-differentiated carcinoma, one moderately differentiated carcinoma, all those three arising de novo and the one arising from an odontogenic cyst[7]. In our case, the lesion was a well differentiated SCC with an advancing edge of moderate differentiation with an absence of any cystic component. Our case was in accordance with almost all the criteria proposed by Suei et al. [4] as there was an absence of any cystic component histologically thus suggesting this case a primary de novo intra osseous SCC.

PIOC is currently managed by wide surgical resection, Neck dissection and reconstruction with vascularized flaps. Radiotherapy is not recommended in view of gross bone involvement. Chemotherapy is indicated either as neoadjuvant therapy if the tumor is inoperable on the first sitting or as an adjuvant therapy if resection margins are positive or if lymph node involvement shows extra-capsular spread [8].

However, the effectiveness of these modalities of treatment is uncertain because of less number of cases and follow-up. The prognosis of this tumor is generally poor. Elzay reported 12 cases of PIOC among which $40 \%$ of patients had successfully completed 2 years survival. Early diagnosis and management eventually yields a better prognosis of these rare tumors.

\section{Conclusion}

This case presented to us with a mild swelling and turned out to be a carcinomatous lesion. Thus, the purpose of reporting this rare tumor is to add existing database that will further service in acquiring knowledge about the origin, behavior, diagnostic imaging, and treatment modalities of this truly uncommon neoplasm. Such cases can pose as diagnostic dilemma and thus the treatment plan should be decided after a biopsy.

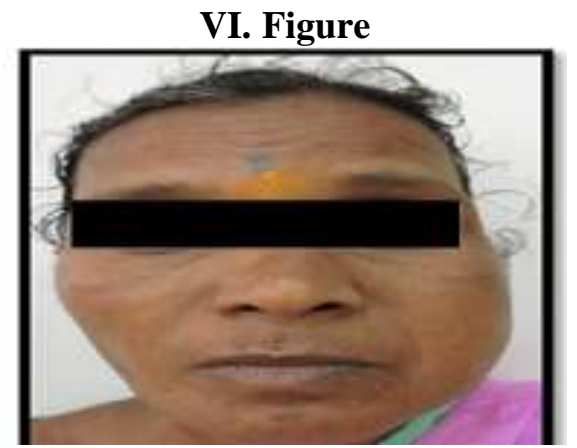

Figure 1 - Clinical picture of swelling present in the left mandibular region causing slight facial asymmetry 


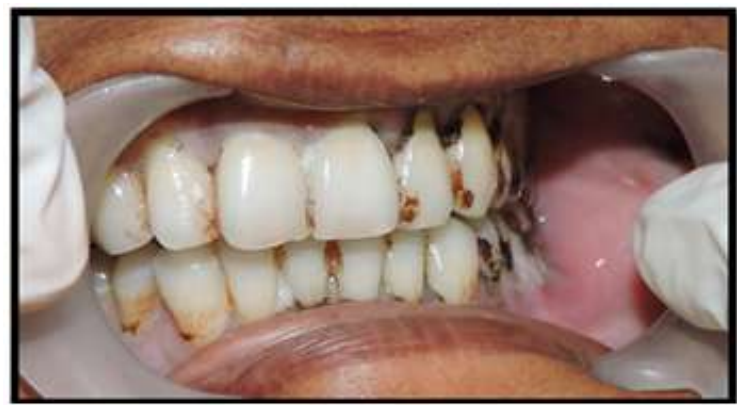

Figure 2 - Intraoral picture showing absence of any defect or proliferative growth

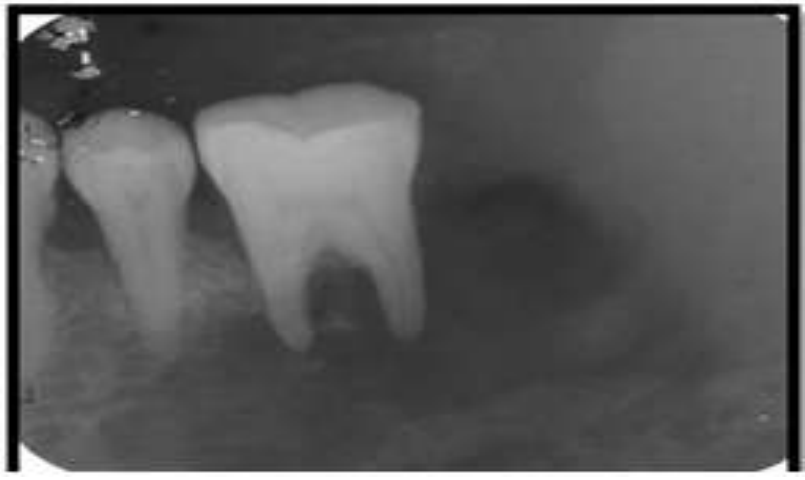

Figure 3 - IOPAR of 35,36 region

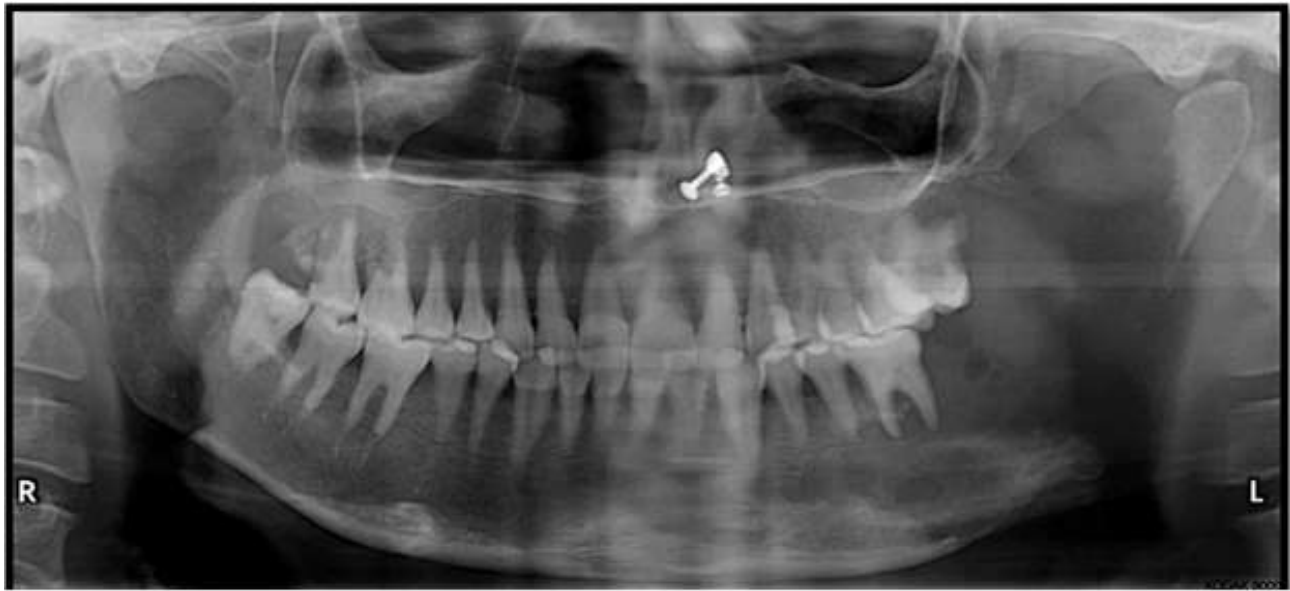

Figure 4 - Orthopantogram shows the presence of large radiolucency in the left mandibular ramus region

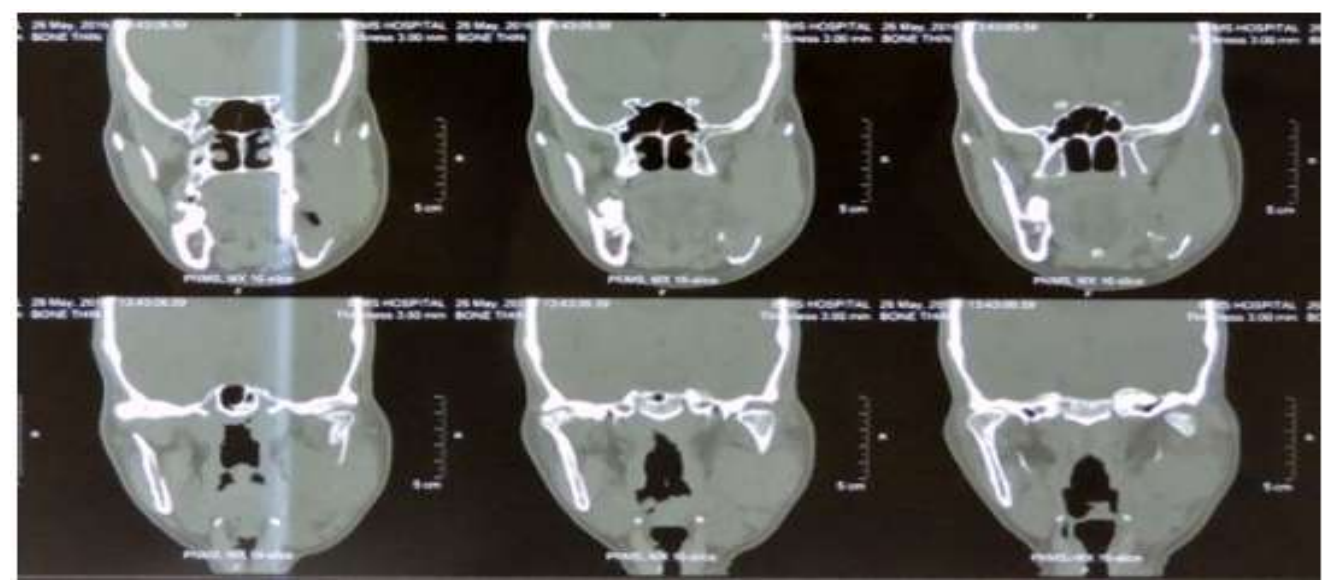

Figure 5 - Coronal view of the CT scan showing the destructive lesion involving the body and ramus of the left half of the mandible 


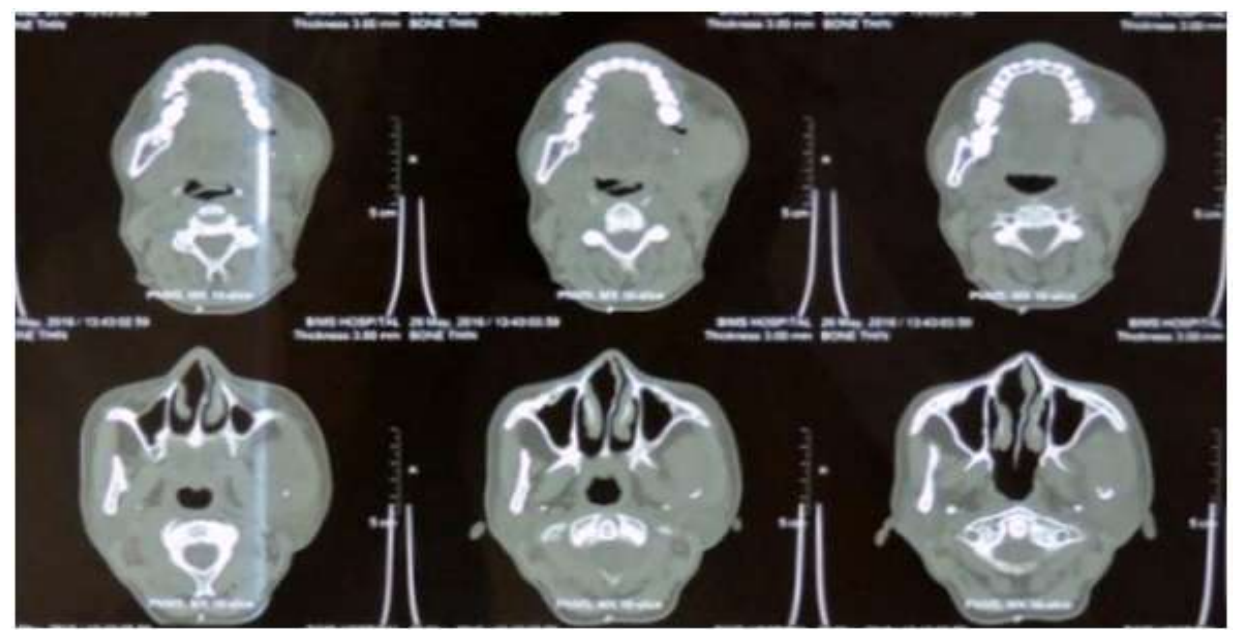

Figure 6 - Axial view of the CT scan showing the destructive lesion involving the body of the left half of the mandible with fragments of condyle and coronoid visible in the sections.

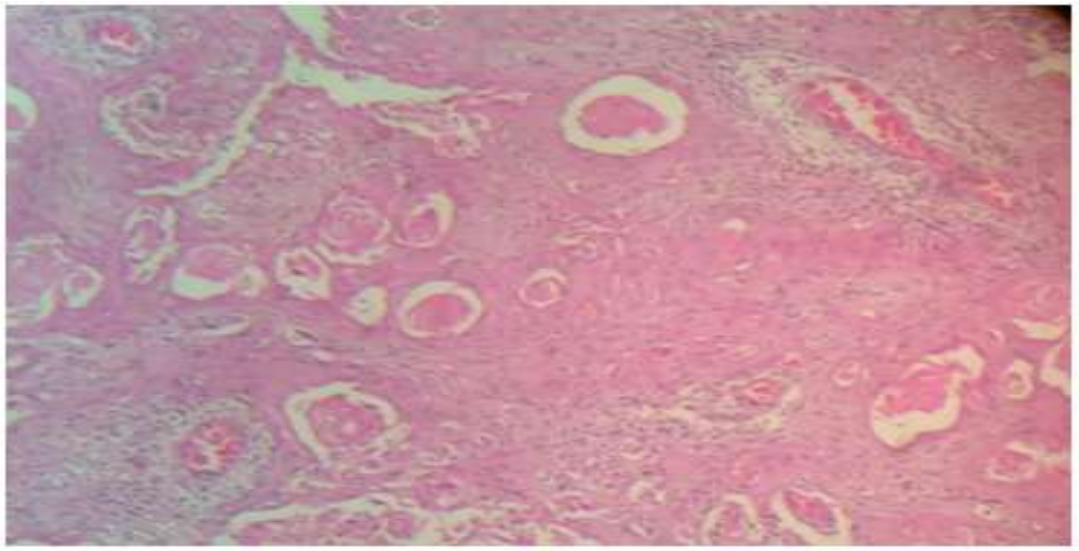

Figure 7 - Histopathological photomicrograph revealing islands of tumor epithelial cells with abundant keratin formation

\section{References}

[1] M Pindborg JJ, Kramer IR, Torloni H. Histological Typing of Odontogenic Tumours, Jaw Cysts, and Allied Lesions. 1st ed. Geneva: World Health Organization; 1991. p. 35-6.

[2] Van Wyk CW, Padayachee A, Nortjé CJ, von der Heyden U. Primary intraosseous carcinoma involving the anterior mandible. Br J Oral Maxillofac Surg 1987;25:427-32.

[3] Anneroth G, Hansen LS. Variations in keratinizing odontogenic cysts and tumors. Oral Surg Oral Med Oral Pathol 1982;54:530-46.

[4] Suei Y, Tanimoto K, Taguchi A, Wada T. Primary intraosseous carcinoma: Review of the literature and diagnostic criteria. J Oral Maxillofac Surg 1994;52:580-3.

[5] Waldron CA, Mustoe TA. Primary intraosseous carcinoma of the mandible with probable origin in an odontogenic cyst. Oral Surg Oral Med Oral Pathol 1989;67:716-24.

[6] Thomas G, Pandey M, Mathew A, Abraham EK, Francis A, Somanathan T, et al. Primary intraosseous carcinoma of the jaw: Pooled analysis of world literature and report of two new cases. Int J Oral Maxillofac Surg 2001;30:349-55.

[7] Yamada T, Ueno T, Moritani N, Mishima K, Hirata A, Matsumura T. Primary intraosseous squamous cell carcinomas: Five new clinicopathologic case studies. J Craniomaxillofac Surg 2009;37:448-53.

[8] Jay S. Cooper et al. Postoperative Concurrent Radiotherapy and Chemotherapy for High-Risk Squamous-Cell Carcinoma of the Head and Neck. N Engl J Med 2004;350:1937-44. 